\title{
ON DISTRIBUTIONS WITH SUPPORT AT THE ORIGIN
}

\section{LEON NOWER ${ }^{1}$}

Among the results of the L. Schwartz theory of distributions is the following

ThEOREM. If $\alpha$ is a q-dimensional distribution whose support is the origin of $R^{q}$, then $\alpha$ is a linear combination of the Dirac $\delta$-distribution and some of its derivatives. [1].

It is the purpose of this paper to supply a new, sequential-theoretic, proof of this theorem. I should like to thank Professor Korevaar for suggesting this problem.

Proof. Let $\left\langle\delta_{N}\right\rangle$ be a fundamental sequence of $C^{\infty}$ functions which defines $\delta$, and such that the support of $\delta_{N}$ is contained in

$$
\begin{array}{r}
I_{N}=\left\{x=\left\langle x_{1}, \cdots, x_{q}\right\rangle \mid-1 / N<x_{i}<1 / N, i=1, \cdots, q\right\}, \\
N=1,2, \cdots .
\end{array}
$$

Then $\alpha_{N}=\alpha^{*} \delta_{N} \in C^{\infty}$, the support of $\alpha_{N}$ is the same as that of $\delta_{N}$, and $\alpha_{N} \rightarrow \alpha$ distributionally. Hence, for some multi-index $m=\left\langle m_{1}, \cdots\right.$, $\left.m_{q}\right\rangle$ there is a sequence $\left\langle G_{N}\right\rangle$ of $C^{\infty}$ functions with the following properties:

(a) $D^{m} G_{N}=\alpha_{N}$ for each $N$; (b) $\left\langle G_{N}\right\rangle$ converges to a continuous function $G$ uniformly on compact sets (and therefore $D^{m} G=\alpha$ ); and, because $I_{N}$ supports $\alpha_{N}$, (c) $G_{N}(x)=0$ if, say, $x_{i}<-1, i=1, \cdots, q$, $N=1,2, \cdots$.

Now let

$$
\begin{aligned}
P(x ; t) & =\prod_{i=1}^{q} \frac{\left(t_{i}-x_{i}\right)^{m_{i}-1}}{\left(m_{i}-1\right) !} \quad \text { if } x_{i}<t_{i}, i=1, \cdots, q, \\
& =0 \quad \text { otherwise, }
\end{aligned}
$$

and

$F_{N}(t)=\int_{-1}^{t_{q}} \ldots \int_{-1}^{t_{1}} D^{m} G_{N}(x) \cdot P(x ; t) d x_{1}, \cdots, d x_{q}, \quad N=1,2, \cdots$.

Integrating by parts $m_{i}$ times with respect to $x_{i}$ and observing that $D^{r} P(x ; t)=0$ if $r_{i}=m_{i}$ for some $i$, we have

Received by the editors March 20, 1967 and, in revised form, June 16, 1967.

1 This research was supported in part by the San Diego State College Foundation. 


$$
\left.F_{N}(t)=\sum_{0<r \leq m}(-1)^{r^{\prime}} D^{m-r} G_{N}(x) \cdot D^{r^{\prime}} P(x ; t)\right]_{x=\{-1, \cdots,-1\rangle}^{x=\left\langle t_{1}, \cdots, t_{q}\right\rangle},
$$

where $(-1)^{r}=\prod_{i=1}^{q}(-1)^{r_{i}}$ and $r^{\prime}=\left\langle r_{1}-1, \cdots, r_{q}-1\right\rangle$. Now, if $r \neq m$ then $D^{r^{\prime}} P(t ; t)=D^{m-r} G_{N}(-1, \cdots,-1)=0$, and if $r=m$, then $D^{r^{\prime}} P(t ; t)=(-1)^{m^{\prime}}$. Hence,

$$
F_{N}(t)=(-1)^{m^{\prime}}(-1)^{m^{\prime}} G_{N}(t)=G_{N}(t) .
$$

Suppose now that $t_{i}<0$ for some $i$. Then for all $N>-1 / t_{i}$ we have $\alpha_{N}(x)=0$ if $x \leqq t$ and $P(x ; t)=0$ otherwise. Hence, $F_{N}(t) \rightarrow 0$. On the other hand, if $t_{i}>0, i=1, \cdots, q$, then for all $N>\max _{i}\left\{1 / t_{i}\right\}$ we have

$$
F_{N}(t)=\int_{-1}^{1 / N} \cdots \int_{-1}^{1 / N} D^{m} G_{N}(x) \cdot P(x ; t) d x_{1} \cdots d x_{q},
$$

and replacing $\left\langle t_{1}, \cdots, t_{q}\right\rangle$ by $\langle 1 / N, \cdots, 1 / N\rangle$ in (1), we see that $F_{N}(t)$ is a polynomial in $t$ of degree $<m_{i}$ in $t_{i}, N=1,2, \cdots$.

Since $F_{N}$ converges to $G$ uniformly on compact sets, it follows that $G$ is equal to a polynomial $F(t)$ of degree $<m_{i}$ in $t_{i}$ on $Q=\left\{t \mid t_{i}>0\right.$, $i=1, \cdots, q\}$, and equal to zero on the complement of $Q$; in other words, $G=U \cdot F$, where $U$ is the characteristic function of $Q$. Therefore,

$$
\alpha=D^{m} G=D^{m}(U \cdot F)=\sum_{0 \leqq r<m}\left(\begin{array}{c}
m \\
r
\end{array}\right) D^{m-r} U \cdot D^{r} F,
$$

where

$$
\left(\begin{array}{c}
m \\
r
\end{array}\right)=\prod_{i=1}^{q}\left(\begin{array}{c}
m_{i} \\
r_{i}
\end{array}\right)
$$

and the strict inequality follows from the fact that $D^{r} F=0$ if $r_{i}=m_{i}$ for some $i$. Now, since for $r<m, D^{r} F$ is a polynomial of degree less than $m_{i}-r_{i}$ in $t_{i}$, and $D^{m-r} U=D^{(m-r)^{\prime}} \delta$, it follows that $D^{m-r} U \cdot D^{r} F$ is a constant multiple of $D^{\left(m-r^{\prime}\right)} \delta$. Thus, (2) may be rewritten as

$$
\alpha=\sum_{0 \leq r_{i}<m_{i}} C_{\left(r_{1}, \cdots, r_{q}\right)} D^{\left(r_{1}, \cdots, r_{q}\right)} \delta .
$$

\section{REFERENCE}

1. L. Schwartz, Théorie des distributions. I, Actualités Sci. Indust. No. 1245, Hermann, Paris, 1957; p. 100.

San Diego State College 